\title{
Programas de Posgrado en Crecimiento Verde y Desarrollo Sostenible: una aproximación comparativa
}

\author{
ALAN FAIRLIE REINOSO* \\ Pontificia Universidad Católica del Perú
}

Recibido el 23-11-2015; primera evaluación el 18-04-2016; segunda evaluación el 25-04-2016; tercera evaluación el 17-05-16; aceptado el 24-05-2016

\section{Resumen}

Dada la preocupación por los impactos negativos que tiene el actual modelo económico en la escasez de recursos naturales y la conservación de estos, nace la necesidad de enfrentarlos y crear, desde la academia, áreas de estudios que contemplen estas nuevas opciones y tendencias de desarrollo. En el presente documento, se realiza una introducción a los aspectos conceptuales de las principales propuestas para la transición hacia el desarrollo sostenible, como son el crecimiento verde, biocomercio, bioeconomía, entre otros. Luego, se identifica y analiza un grupo de programas de posgrado desarrollados en las líneas de desarrollo sostenible. Por último, se consolida un balance comparativo sobre la base de la oferta de la Maestría de Biocomercio y Desarrollo Sostenible.

Palabras clave: desarrollo sostenible, crecimiento verde, posgrado, enfoques de programa, planes de estudio.

\footnotetext{
* Licenciado en Economía en la Pontificia Universidad Católica del Perú. Magíster en Comercio Internacional y Desarrollo en el Instituto Torcuato Di Tella en Buenos Aires, Argentina. Magister en Política y Gestión Universitaria en la Universidad de Barcelona, Espańa. Actualmente, decano de la Facultad de Ciencias Sociales de la Pontificia Universidad Católica del Perú y director de la Maestría en Biocomercio y Desarrollo Sostenible. Coordinador del Área Andina para RED LATN. Consultor internacional con diversas publicaciones sobre integración económica, negociaciones comerciales internacionales, biocomercio y crecimiento verde. Autor de La Alianza del Pacifico: una mirada desde el Perú (en edición), Integración y convergencia en UNASUR (Red LATN, 2013). Editor del libro Los paises andinos frente al TLC y la Comunidad Sudamericana de Naciones, coautor del libro Cadenas productivas y clisters en el sector gastronómico peruano (2012), Biocomercio en el Perú: experiencias y propuestas (2010) y Competitividad e integración: las pymes industriales exportadoras del Perú (2003). Contacto: afairli@pucp.edu.pe
} 
Graduate Programs in Green Growth and Sustainable Development: A Comparative Approach

\section{Abstract}

Since the concern about the negative impacts of the current economic model in the scarcity of natural resources and their conservation, it comes the need to confront them and create, from academia, some research areas that include these new features and development trends. In this paper, an overview of the conceptual aspects of the main proposals for the transition to sustainable development is performed, such as the Green Growth, Bio-Commerce, Bio-Economy, among others. Then, it is identified and analyzed a group of graduate programs developed on the sustainable development. Finally, a comparative balance, based on the offer of the Master of Bio and Sustainable Development.

Keywords: sustainable development, green growth, post grade, program approaches, curricula.

Pós-Graduação em Crescimento Verde e Desenvolvimento Sustentável: uma abordagem comparativa

\section{Resumo}

Dada a preocupação com os impactos negativos do atual modelo econômico na escassez de recursos naturais e à conservaçáo destes, vem a necessidade de enfrentá-los e criar, a partir de universidades, áreas de pesquisa que incluem esses novos recursos e tendências de desenvolvimento. Neste trabalho, uma visão geral dos aspectos conceituais das principais propostas para a transiçáo para o desenvolvimento sustentável é realizada. o crescimento verde, Bio-Comércio, Bio-Economia, entre outros. Em seguida, identifica e analisa um grupo de programas de pós-graduação desenvolvidos sobre as linhas de desenvolvimento sustentável. Finalmente, um balanço consolidado comparativo baseado na oferta do Mestrado em Bio e Desenvolvimento Sustentável.

Palavras-chave: desenvolvimento sustentável, crescimento verde, abordagens do programa de pós-graduação, currículos. 


\section{BIOECONOMÍA, BIOCOMERCIO Y CRECIMIENTO VERDE ${ }^{1}$ : ASPECTOS CONCEPTUALES}

Existen propuestas que buscan ser alternativas al actual modelo económico global. Entre ellas destaca la Iniciativa de Economía Verde que nace por parte del Programa de las Naciones Unidas para el Medio Ambiente, Crecimiento Verde y Desarrollo Sostenible lanzada por la Organización para la Cooperación y el Desarrollo Económicos (OECD) (2011); el Biocomercio (principios y criterios) fue desarrollado por la Conferencia de las Naciones Unidas sobre Comercio y Desarrollo (UNCTAD, por sus siglas en inglés) (2007); y el Crecimiento Verde Inclusivo planteado por el Banco Mundial. Adicionalmente, desde el Sistema Económico Latinoamericano y del Caribe (SELA) se han sostenido lineamientos con una perspectiva latinoamericana.

El concepto de economía verde, desarrolla propuestas sobre políticas consideradas relevantes para alcanzar el desarrollo sostenible. Una economía verde es aquella que genera bajas emisiones de carbono, hace uso de los recursos de forma eficiente y es inclusiva socialmente.

El crecimiento verde tiene como pilares la inversión y la innovación como medios para consolidar el crecimiento sostenido y generar nuevas oportunidades económicas. Adicionalmente, requiere de una utilización eficiente de los recursos para minimizar las presiones ambientales. Los medios que propone son estímulos de políticas para promover la productividad, innovación, y nuevos mercados a la vez que plantean estrategias que favorezcan la reducción de impactos negativos a nivel social y ambiental.

El SELA tiene una propuesta de economía verde redistributiva, donde interactúen los agentes y factores de producción, de manera tal que permita alcanzar un desarrollo incluyente. También, se debe respetar los derechos de los países menos desarrollados, teniendo un trato diferenciado en la transición hacia una economía verde. Asimismo, establecer un valor a los bienes y servicios del capital natural.

El biocomercio postula principios y criterios de sostenibilidad ambiental, social y económica que deben ser internalizados en las diferentes fases de producción hasta la comercialización de bienes y servicios, los cuales involucran prácticas de conservación y uso sostenible.

Por último, la bioeconomía es definida como la economía en la cual el consumo y producción de bienes y servicios, están basados directamente en el uso y transformación de los recursos biológicos y del uso productivo de

1 Esta sección se basa en Fairlie (2013). 
los desperdicios generados del proceso de consumo y producción. La bioeconomía incluye tecnologías que son usadas en la transformación de los recursos biológicos, en especial, tecnologías relacionadas a la biotecnología.

Para alcanzar el desarrollo de la bioeconomía se propone impulsar el desarrollo del recurso humano y optimizar los mecanismos para la participación social. De acuerdo a esto, la bioeconomía puede incluir tres elementos: el uso de un conocimiento avanzado de los genes y procesos celulares complejos para desarrollar nuevos procesos y productos. Además, la utilización de biomasa renovable y bioprocesos eficientes para apoyar la producción sostenible; y, la integración de los conocimientos de biotecnología y aplicaciones a través de sectores.

La OECD como el Banco Mundial y el Programa de Naciones Unidas para el Medio Ambiente (PNUMA) subrayan que sus propuestas de "Crecimiento verde», "Crecimiento verde sustentable» $\mathrm{y}$ «Economía verde» complementan la propuesta de «Desarrollo sustentable» y no la reemplazan, reconocen que es indispensable la sustentabilidad para enverdecer la economía.

Sin embargo, existen una serie de posturas críticas a estos nuevos modelos; al igual que otras propuestas alternativas. Fairlie (2013) realiza un balance sobre aproximaciones críticas hacia las propuestas señaladas líneas arriba.

Una de las críticas al modelo de economía verde es que sigue basándose en el crecimiento económico, sin tomar en cuenta los límites del planeta. Por ende, esta propuesta se basa en una sostenibilidad débil. La economía verde debe resolver el problema de la desigualdad y no reproducir un patrón comercial norte-sur.

Respecto al objetivo de reducción de la pobreza, se señala que el principal problema es que apoya la liberalización comercial; con lo cual se acentúan las ideas de especialización productiva y fomenta las relaciones norte-sur, lo cual se considera no permite la convergencia entre países.

Respecto al crecimiento verde se presenta como la opción ideal. Las pautas, medidas y políticas que se deben seguir para construir esta economía pueden implicar algunos costos en el corto plazo sobre el consumo, productividad y bienestar. Asimismo, obtener resultados variados de acuerdo a la coyuntura de cada país donde se aplique (Da Motta \& Polónia, 2013).

En esta línea, Hallegatte, Heal, Fay y Treguer (2012) plantean que las políticas ambientales pueden generar costos en el corto plazo que pueden crear un trade-off con el crecimiento económico. No existe una asociación automática entre crecimiento verde y el impacto sobre los mercados de trabajo, que permita una reducción de la pobreza. Esto puede complicar el avance hacia un crecimiento verde inclusivo. 
Una preocupación está referida a la capacidad local de nuevas tecnologías y la dependencia potencial que se pueda generar. Se requieren cambios estructurales en los sectores productivos para lograr un crecimiento verde, lo cual no es una tarea de corto plazo (Cosbey, 2011; Ocampo, 2011).

Khor (2011) identifica ciertos riesgos en el concepto de economía verde: tendencia a uniteralizar lo ambiental, a tratar a todos los países como iguales, y el uso de los estándares ambientales como nuevas medidas de protección ambiental.

Otros autores atribuyen una visión economicista al concepto de crecimiento verde, y desde la economía ecológica cuestionan lo que ellos consideran limitaciones de un enfoque de economía ambiental neoclásica (Martínez Alier $\&$ Roca, 2001). Se debe considerar la sostenibilidad fuerte, que parte de la no sustituibilidad entre el capital natural y el capital fabricado (Neumayer, 2003).

En la siguiente sección se hace una primera aproximación a programas de posgrado que abordan esta problemática, buscando sistematizar y comparar sus enfoques.

\section{ForMACIÓN DE POSGRADO EN CRECIMIENTO VERDE}

En el presente apartado se hace un balance de diferentes programas de posgrado que están orientados a tratar los siguientes temas:

1. Bioeconomía y biotecnología

2. Desarrollo sostenible

3. Gestión ambiental

4. Medio ambiente, recursos naturales y biodiversidad

5. Gestión de energía

Luego de una búsqueda a través de las páginas web de universidades de Latinoamérica y otras regiones, se encontraron diversas maestrías enfocadas en la formación de profesionales en temas de desarrollo sostenible, bioeconomía y temas relacionados. A continuación se presenta una primera sistematización de sus objetivos.

\subsection{Bioeconomía y biotecnología}

En Latinoamérica, existen ciertas maestrías que impulsan la bioeconomía. Se encontró cuatro maestrías orientadas a la formación de profesionales en biotecnología y una orientada a la tecnología ambiental. La mayoría de los objetivos de estas maestrías proponen tener un impacto en el sector agrario e industrial 
a través de la investigación científica. Las maestrías son las siguientes: Maestría de Ciencias en Biotecnología de la Universidad Nacional de Colombia, Maestría en Biotecnología de la Universidad ICESI, la Maestría en Biotecnología Industrial de la Universidad Positivo de Brasil, Maestría de Investigación en Ciencias en Biotecnología Agrícola del Centro de Investigaciones Biotecnológicas del Ecuador; y, por último, la Maestría en Tecnologías Ambientales dictada por la Universidad Federal Tecnológica de Paraná de Brasil.

La sede en Medellín de la Universidad Nacional de Colombia ofrece la Maestría de Ciencias en Biotecnología. Esta maestría tiene el objetivo de formar talento humano calificado en fundamentos básicos de la biología celular y molecular, la bioquímica, la biofísica y la estadística, con el fin de impulsar el desarrollo en las áreas vegetal, microbiana, animal e industrial.

La Universidad ICESI ubicada en Cali en Colombia, propone su Programa de Posgrado en Biotecnología, cuyo objetivo general es que los profesionales adquieran los fundamentos técnico-científicos interdisciplinarios para liderar procesos de investigación-desarrollo-innovación $(\mathrm{I}+\mathrm{D}+\mathrm{i})$ en biotecnología. Las áreas de concentración para investigación de la maestría son: biotecnología agrícola, molecular, celular y de organismos; biotecnología ambiental y bioindustrial; biotecnología farmacéutica y biotecnología médica.

La Maestría en Biotecnología Industrial de la Universidad Positivo de Brasil tiene como fin formar maestros en Biotecnología Industrial y proporcionar las condiciones para que los graduados ya puedan actualizarse científica, pedagógica y profesionalmente. Para ello, el estudiante profundiza el conocimiento a través de las disciplinas estructurales y el desarrollo de un innovador.

Asimismo, el Centro de Investigaciones Biotecnológicas del Ecuador ofrece una Maestría en Biotecnología especializada en el sector agrícola, que busca formar profesionales investigadores para transferir al sector agrícola las oportunidades que a través de investigación básica, aplicada y participativa, contribuyan al incremento de la productividad y el establecimiento de sistemas de producción agrícola sostenible y ambientalmente amigable.

Por último, la Maestría en Tecnologías Ambientales de la Universidad Federal Tecnológica de Paraná de Brasil, tiene como objetivo la formación de profesionales calificados para trabajar en el marco de la investigación y la enseñanza, así como la búsqueda de soluciones innovadoras a fin de conciliar el desarrollo económico y social de la conservación de los recursos naturales.

En Europa se observa una mayor oferta de maestrías especializadas en el tema de bioeconomía que incorporan en sus planes de estudio metodologías biotecnológicas. En este caso, se identificó tres maestrías: la Maestría en Gestión de la Bioeconomía, Innovación y Gobernanza de la Universidad 
de Edimburgo en Escocia, la Maestría en Bioeconomía de la Universidad de Hohenheim de Alemania y la Maestría en Gestión en Bioeconomía de la Universidad de Lovaina de Bélgica.

La Maestría en Gestión de la Bioeconomía, Innovación y Gobernanza de la Universidad de Edimburgo en Escocia, desde un enfoque interdisciplinario, busca permitir a los graduados sobresalir en el pensamiento estratégico y crítico que aporta soluciones contextualizadas a nivel mundial a los problemas prácticos relacionados con: la innovación y la estrategia de la empresa, la política y la regulación, la investigación en colaboración, el desarrollo, la gobernabilidad y la propiedad intelectual.

Del mismo modo, la Maestría en Bioeconomía de la Universidad de Hohenheim de Alemania pretende fomentar el pensamiento crítico. Los graduados tienen las habilidades necesarias para desarrollar y ejecutar diversas actividades dentro de una economía de base biológica.

Por último, la Maestría en Gestión en Bioeconomía de la Universidad de Lovaina de Bélgica tiene como objetivo dar una introducción de los conceptos y técnicas de gestión fundamentales aplicados a la bioeconomía, que es el conjunto de actividades económicas relacionadas con la invención, el desarrollo, la producción y el uso de productos y procesos biológicos.

\subsection{Desarrollo sostenible}

Existe una gran oferta de programas de posgrado en Latinoamérica dirigidos a la formación de profesionales en el tema de desarrollo sostenible. Se pueden identificar las maestrías vinculadas a temas de desarrollo sostenible en el sector agrario y, otras que tienen objetivos como la calidad de vida o conservación de recursos biológicos.

Las maestrías son: Maestría en Biocomercio y Desarrollo Sostenible de la Pontificia Universidad Católica del Perú, Maestría en Desarrollo Sostenible de Sistemas Agrarios de la Pontificia Universidad Javeriana de Colombia, Maestría en Agroecología y Desarrollo Sostenible de la Universidad Autónoma Gabriel René Moreno en Bolivia, Magíster en Agricultura Ecológica de la Universidad Nacional de Costa Rica, Maestría en Desarrollo Sostenible de Universidad de Brasilia en Brasil, Maestría en Altos Estudios Amazónicos de la Pontificia Universidad Católica del Perú, Maestría en Desarrollo Sostenible y Calidad de Vida del Centro Universitario de los Colegios Asociados (UniFAE) São João da Boa Vista de Brasil y, por último, Maestría Académica en Desarrollo Sostenible con énfasis en Conservación de los Recursos Biológicos de la Universidad Autónoma Gabriel René Moreno de Bolivia. Asimismo, se 
presenta el diploma superior sobre Derecho y Economía del Cambio Climático que brinda FLACSO-Sede Argentina.

La Maestría de Biocomercio y Desarrollo Sostenible ofrecida por la Pontificia Universidad Católica del Perú (PUCP) es un programa interdisciplinario que plantea que el biocomercio es una opción de crecimiento verde. El enfoque del programa es desarrollado por el plan curricular y los contenidos de los cursos, donde se atiende trasversalmente a los criterios de sostenibilidad social, económica y ambiental.

Es así que el programa aborda la importancia del biocomercio en las regiones andinas y el desarrollo sostenible como tema trascendental a nivel mundial. Básicamente, se trata de ponderar el concepto de biocomercio que vincula investigación en torno principalmente a planes de negocios, competitividad, cadenas productivas y asociatividad.

Las líneas temáticas en las que se enmarcan los cursos son: elaboración de planes de bionegocios de bienes y servicios de biodiversidad, factores determinantes de la competitividad en bionegocios, planificación territorial con criterios de desarrollo sostenible, desarrollo de cadenas productivas basadas en principios de biocomercio, y estudios de asociatividad en organización de productores e impacto de los sellos de comercio justo y orgánico.

El objetivo de la maestría es brindar una oferta académica de tipo profesional sobre el sistema de biocomercio de recursos biológicos nativos, que se basa fundamentalmente en la articulación de la sostenibilidad económica, social y ambiental como partes indesligables del desarrollo sostenible. La oferta pretende estar en línea con la tendencia de las universidades mundiales de ofrecer programa sobre desarrollo sostenible para satisfacer la creciente demanda a este respecto.

La Maestría en Desarrollo Sostenible de Sistemas Agrarios de la Pontificia Universidad Javeriana de Colombia busca formar a los estudiantes en los fundamentos científicos de los sistemas productivos agropecuarios sostenibles y adiestrados en el manejo del conjunto de técnicas que los hacen viables. Al mismo tiempo, se propone formar profesionales que tengan la capacidad de identificar e investigar limitantes del desarrollo rural y las alternativas de superación de los mismos. Para ello se vincula al estudiante a un proceso de construcción de soluciones productivas viables por medio de la investigación y la validación de tecnologías - tanto en granja experimental como con los productores y con las comunidades rurales-, difusión y extensión de las mismas.

La Maestría en Agroecología y Desarrollo Sostenible de la Universidad Autónoma Gabriel René Moreno en Bolivia tiene como finalidad lograr que 
los profesionales adquieran e integren conocimientos y habilidades de la ciencia y técnica agrícola apropiados a las condiciones del país, así como el de los componentes socioeconómicos y ecológicos de los sistemas agropecuarios, de tal manera que; aporten al desarrollo agrícola verdaderamente sostenible, que satisfaga las necesidades actuales y futuras de la población, sin degradar el medio ambiente.

La Maestría en Agricultura Ecológica de la Universidad Nacional de Costa Rica busca tener una mayor profundización en el análisis de los sistemas de producción y en la generación de opciones de solución a los problemas del impacto de la producción agrícola sobre el ambiente, con lo cual se potenciará la actividad investigativa en este campo.

La Maestría en Desarrollo Sostenible de la Universidad de Brasilia en Brasil posee tres objetivos:

- Producir información y conocimiento que contribuye a la esclarecimiento, diagnóstico, cuantificación, seguimiento y prevención de daños al medio ambiente

- Desarrollar metodologías y enfoques interdisciplinarios para la integración de profesionales de diversos orígenes disciplinarios en los equipos de expertos para abordar los problemas ambientales complejos

- Contribuir, a través de la formación de recursos humanos de alto nivel, al fortalecimiento institucional de los organismos y organizaciones que trabajan en la ciencia y la tecnología ambiental

Del mismo modo, la Maestría en Desarrollo Sostenible y Calidad de Vida del Centro Universitario de los Colegios Asociados (UniFAE) São João da Boa Vista de Brasil busca formar profesionales capaces de crear y difundir modelos innovadores de desarrollo sostenible. Además, generar conocimiento para intervenir en la realidad regional y proporcionar formación interdisciplinaria actualizada en el desarrollo sostenible y las prácticas de comunicación para la promoción de la calidad de vida.

FLACSO-Sede Argentina ofrece el diploma superior sobre Derecho y Economía del Cambio Climático relacionado con las cuestiones de comercio y medio ambiente. Este parte de la problemática del cambio climático para brindar una enseñanza interdisciplinaria que adiestre en la toma de decisiones en un escenario de cambio global.

La especialización en Derecho y Cambio Climático está organizada alrededor de materias tales como economía ambiental, derecho internacional del cambio climático, economía de la energía, etc. 
La Maestría Académica en Desarrollo Sostenible con énfasis en Conservación de los Recursos Biológicos de la Universidad de Costa Rica busca formar investigadores, docentes y profesionales comprometidos con la sostenibilidad de los recursos naturales, capaces de proponer alternativas para la prevención y solución de los problemas ambientales, la planificación urbana y el desarrollo sostenible.

Asimismo, se identificaron tres maestrías orientadas a promover el desarrollo sostenible desde el sector académico a otros sectores como el sector público, el sector empresarial, etc. Estas maestrías son las siguientes: la Maestría en Sostenibilidad e Innovación Social de la Escuela de Estudios Superiores de Comercio (HEC) de París, la Maestría en Desarrollo Sostenible de la Universidad de Uppsala y la Universidad Sueca de Ciencias Agrícolas en Suecia y, finalmente, la Maestría en Medio Ambiente y Desarrollo Sostenible de la Escuela de Negocios de Adam Smith de la Universidad de Glasgow en el Reino Unido.

La Maestría en Sostenibilidad e Innovación Social de la HEC de París (Escuela de Estudios Comerciales) busca que los estudiantes logren crear modelos de negocio más ecológicos y sostenibles, e incorporen estrategias de desarrollo sostenible.

La Maestría en Desarrollo Sostenible de la Universidad de Uppsala y la Universidad Sueca de Ciencias Agrícolas tiene como objetivo permitir utilizar los aspectos del desarrollo sostenible en la futura actividad profesional, esta podría ser la planificación social, la educación, la investigación, la organización de los organismos supranacionales, políticas sobre el futuro de la biodiversidad, y mucho más.

La Maestría de Altos Estudios Amazónicos de la Pontificia Universidad Católica del Perú busca dar una formación integral creando espacios en los que se pueda responder a los retos que supone abordar una realidad tan amplia y compleja como la de selva amazónica, la cual, reconocida por su riqueza genética, además de la diversidad cultural y lingüística, constituye las dos terceras partes del territorio nacional. Asimismo, el programa pretende contribuir al fortalecimiento de la institucionalidad regional frente al desarrollo humano sostenible.

Por último, la Maestría en Medio Ambiente y Desarrollo Sostenible de la Escuela de Negocios de Adam Smith de la Universidad de Glasgow en el Reino Unido, busca enseñar herramientas a los estudiantes para aplicar el análisis económico para un rango de problemas de gestión ambiental con un enfoque en los países en desarrollo. 


\subsection{Gestión ambiental}

En Latinoamérica, identificamos cinco maestrías respecto a gestión ambiental dirigidas básicamente manejar el tema sostenibilidad desde la perspectiva empresarial. Las maestrías son las siguientes: la Maestría en Gestión ambiental de la Universidad de los Andes en Colombia, el MBA ${ }^{2}$ en Gestión Ambiental y Desarrollo Sostenible de la Universidad Federal de Juiz de Fora en Brasil, el $\mathrm{MBE}^{3}$ en Economía y Gestión Sostenible de la Universidad Federal de Rio de Janeiro dictada por Instituto de Economía en Brasil, Maestría en Gestión de los Recursos Hídricos y la Maestría en Desarrollo Ambiental; estas últimas de la Pontificia Universidad Católica del Perú.

La Maestría en Gestión Ambiental de la Universidad de los Andes en Colombia tiene como objetivo desarrollar habilidades y competencias gerenciales para liderar procesos de cambio a partir de los retos y oportunidades socio-ambientales de las organizaciones. Esta maestría busca tener una perspectiva crítica e interdisciplinaria en el marco del desarrollo sostenible.

El MBA en Gestión Ambiental y Desarrollo Sostenible de la Universidad Federal de Juiz de Fora en Brasil, posee el objetivo de formar profesionales competentes para el ejercicio de actividades relacionadas con el medio ambiente, dándoles la posibilidad de poner en práctica programas de planificación y gestión ambiental en las empresas públicas y privadas. En general, esta maestría pretende que el especialista pueda actuar de una manera objetiva y sistemática en la búsqueda de soluciones a los problemas ambientales globales y regionales.

El Programa de Posgrado en Desarrollo Ambiental de la Pontificia Universidad Católica del Perú, desde un enfoque interdisciplinario, busca formar profesionales que sean capaces de contribuir al logro de un desarrollo sostenible y armónico al vincular el desarrollo humano con el ecológico; de esta manera quiere fortalecer capacidades de los sectores tanto público como privado para la solución de problemas ambientales.

Por su parte, la Maestría en Gestión de Recursos Hídricos tiene como fin brindar al alumno los conocimientos, competencias y habilidades para comprender y analizar los principios que regulan la gestión del agua en la región andina y que en su desempeño profesional pueda proponer soluciones a los problemas vinculados con la gestión del agua.

\footnotetext{
2 Maestría en Administración de Negocios o Máster en Administración y Dirección de Empresas (Master of Business Administration en inglés, abreviado MBA).

3 Máster de Ingeniería de Negocios (Master of Business Engineering en inglés, abreviado MBE).
} 
Por último, el MBE en Economía y Gestión Sostenible de la Universidad Federal de Rio de Janeiro dictada por el Instituto de Economía en Brasil tiene como propósito formar a los directivos y proporcionar capacitación al personal a nivel de posgrado con amplio sentido en economía y gestión de la sostenibilidad.

En los países de Italia, Canadá, Reino Unido y Australia diversas universidades ofertan maestrías relacionadas a la gestión ambiental, este enfoque trata de manejar los problemas ambientales y de sostenibilidad desde el punto de vista del sector privado. Se encontraron cuatro maestrías relacionadas a este tema: la Maestría en Gestión Ambiental, Energía y Responsabilidad Social Corporativa de la Universidad de Bocconi de Italia, la Maestría en Gestión Ambiental para Negocios de la Universidad de Cranfield del Reino Unido, la Maestría en Ciencias en Estudios de Gestión de Recursos y Medio Ambiente de la Universidad de British Columbia en Canadá y, finalmente, la Maestría en Gestión Ambiental de la Universidad de Queensland de Australia.

La Maestría en Gestión Ambiental, Energía y Responsabilidad Social Corporativa de la Universidad de Bocconi de Italia tiene como objetivo proporcionar a los estudiantes conciencia y conocimiento de la teoría, regulación y aspectos éticos del tema medio ambiental y, además, de la enseñanza de las habilidades avanzadas de gestión y competencia.

La Maestría en Gestión Ambiental para Negocios de la Universidad de Cranfield del Reino Unido busca formar un nuevo tipo de profesional con visión, habilidades, conocimientos y capacidad para enfrentar el reto de cambiar los patrones de producción y consumo, para lograr objetivos de desarrollo sostenible sin comprometer la viabilidad futura de los sistemas naturales y humanos.

La Maestría en Ciencias en Estudios de Gestión de Recursos y Medio Ambiente de la Universidad de British Columbia en Canadá, tiene como fin promover un futuro sostenible mediante la investigación integrada y el aprendizaje sobre los vínculos entre humanos y los sistemas naturales, y para apoyar la toma de decisión a escala local y global.

Por último, el objetivo de la Maestría en Gestión Ambiental de la Universidad de Queensland en Australia, es producir gerentes capaces de hacer frente a los muchos problemas en la zona altamente compleja y cambiante de la gestión ambiental.

\subsection{Medio ambiente, recursos naturales y biodiversidad}

Hay otras maestrías que tienen como perspectiva el tema de conservación ambiental y manejo de recursos naturales como la biodiversidad, incorporando 
temas de sostenibilidad. En América Latina se consideraron tres maestrías que siguen esta perspectiva: la Maestría en Medio Ambiente y Desarrollo de la Escuela de Ciencias Económicas de la Universidad Nacional de Colombia, la Maestría en Biodiversidad y Gestión de Ecosistemas de la Universidad Nacional Mayor de San Marcos de Perú y, en último lugar, la Maestría en Conservación y Uso de Biodiversidad de la Pontificia Universidad Javeriana de Colombia.

La Maestría en Medio Ambiente y Desarrollo de la Escuela de Ciencias Económicas de la Universidad Nacional de Colombia, tiene un enfoque interdisciplinario basado en la conjunción de la escuela de leyes, de política y de ciencias sociales. Su objetivo principal es promover en los estudiantes la capacidad de comprender y analizar estas relaciones complejas, incorporando en el análisis variables del entorno de desarrollo y ecoculturales. Asimismo, trabajar para el uso sostenible y la conservación del medio ambiente.

La Maestría en Biodiversidad y Gestión de Ecosistemas de la Universidad Nacional Mayor de San Marcos de Perú persigue el objetivo de conocer aspectos teóricos y prácticos de la diversidad animal y de la diversidad vegetal y los conocimientos y experiencia necesarias para que se incorpore al ámbito laboral. Además, que el profesional tengan impacto en las instituciones para que desarrollen inventarios de la biodiversidad, desarrollo de planes territoriales, auditoría ambiental, diseńo y gestión de áreas protegidas entre otros.

La Maestría en Conservación y Uso de Biodiversidad de la Pontificia Universidad Javeriana de Colombia, busca formar profesionales con una base sólida de conocimientos y competencias sobre los enfoques, conceptos y métodos en el campo de la conservación y uso de la biodiversidad.

En Estados Unidos y en Reino Unido existen dos maestrías que desarrollan temas del medio ambiente desde una perspectiva menos económica. Este es el caso de la Maestría en Medio Ambiente y Desarrollo de las Universidad de Stanford en Estados Unidos y la Escuela de Economía y Ciencia Política de Reino Unido, respectivamente. Estas se orientan a una formación interdisciplinar, en la cual,la dimensión económica tiene igual relevancia que otras.

La Maestría en Medio Ambiente y Recursos de la Universidad Stanford en Estados Unidos tiene como objetivo el desarrollo en los estudiantes de conocimientos, habilidades, perspectivas y formas de pensar para entender y resolver los desafíos medioambientales y sostenibilidad de recursos.

La Maestría en Medio Ambiente y Desarrollo de la Escuela de Economía y Ciencia Política de Londres, propone proporcionar a los estudiantes una formación rigurosa en las dimensiones de ciencias sociales de la política ambiental y el desarrollo, gestión y evaluación dentro de un contexto de país en desarrollo. 


\subsection{Gestión de energía}

En el Perú identificamos dos propuestas de posgrado sobre el manejo y la gestión del sector energético, las dos maestrías son las siguientes: el MBA en Energía de Centrum Católica y la Maestría de la Gestión de la Energía de la Universidad ESAN.

El MBA en Energía de Centrum Católica de Perú es una opción de especialización no funcional del programa de MBA de Centrum. Este programa busca formar a ejecutivos que tengan interés en el sector energético en temas de gestión de energía, del sector minero y, a la vez, incluir aspectos ambientales.

El objetivo de la Maestría de la Gestión de la Energía de la Universidad ESAN es ofrecer una formación integral en gestión de la energía con especializaciones en el sector eléctrico e hidrocarburos y gas que profundicen los conocimientos de tipo económico, regulatorio y financiero que se requieren en la gestión técnica y económica de las distintas áreas de negocio en las empresas del sector energía.

En resumen, los programas arriba descritos, poseen ciertos criterios en los que convergen. Los programas en temas de desarrollo sostenible, medioambiente, recursos naturales y biodiversidad y de gestión ambiental son interdisciplinarios, mientras que el resto son de corte especializado. Asimismo, los principales cursos que comparten su currícula son: economía, gestión y biología. Se hace un balance comparativo en la siguiente sección.

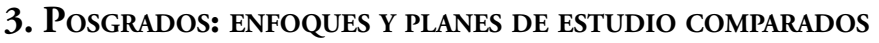

De la muestra de maestrías orientadas a los temas antes mencionados, se encontró que de un total de 36 programas, casi el $75 \%$ de ellas proponen un enfoque interdisciplinario y cerca del $25 \%$ propone un enfoque especializado.

En el caso de programas latinoamericanos, de un total de 24 , solo 6 tienen un enfoque disciplinario y 18 maestrías tienen una visión interdisciplinaria. Por una parte, los programas de posgrado de Latinoamérica que tienen visión disciplinaria son orientados a desarrollar principalmente temas de biotecnología, gestión y gestión de energía. Estos programas son: Maestría en Ciencias Biotecnología de la Universidad Nacional de Colombia, Maestría de Investigación en Ciencias en Biotecnología Agrícola del Centro de Investigaciones Biotecnológicas del Ecuador, Maestría en Biotecnología de la Universidad ICESI de Colombia, la especialización MBA en Energía de Centrum Católica de Perú, la Maestría de la Gestión de la energía de la Universidad ESAN en Perú y, por último, el MBA en Gestión Ambiental y 
Desarrollo Sostenible de la Universidad Federal de Juiz de Fora de Brasil. Por otra parte, las maestrías orientadas con visión interdisciplinaria, forman principalmente estudiantes en temas de desarrollo sostenible, gestión ambiental, medio ambiente y recursos naturales y una en biotecnología industrial.

\section{Cuadro 1. Maestrías con enfoque interdisciplinario}

\begin{tabular}{|c|c|}
\hline Maestría & Universidad \\
\hline $\begin{array}{l}\text { Maestría en Biocomercio y Desarrollo } \\
\text { Sostenible }\end{array}$ & Pontificia Universidad Católica del Perú \\
\hline $\begin{array}{l}\text { Magíster en Desarrollo Sostenible de } \\
\text { Sistemas Agrarios }\end{array}$ & Pontificia Universidad Javeriana, Colombia \\
\hline $\begin{array}{l}\text { Maestría en Desarrollo Sostenible y Calidad } \\
\text { de Vida }\end{array}$ & UniFAE Brasil \\
\hline Maestría en Desarrollo Sostenible & Universidad de Brasília, Brasil \\
\hline $\begin{array}{l}\text { Maestría en Desarrollo Sostenible con énfasis } \\
\text { en Conservación de los Recursos Biológicos }\end{array}$ & Universidad de Costa Rica \\
\hline Magíster en Agricultura Ecológica & Universidad Nacional de Costa Rica \\
\hline Maestría en Gestión Ambiental & Universidad de los Andes, Colombia \\
\hline MBE en Economía y Gestión Sostenible & $\begin{array}{l}\text { UFRJ, Universidad Federal de Rio de } \\
\text { Janeiro, Instituto de Economía }\end{array}$ \\
\hline Master in Environment and Development & $\begin{array}{l}\text { School of Economic Sciences, Universidad } \\
\text { Nacional de Colombia }\end{array}$ \\
\hline $\begin{array}{l}\text { Maestría en Biodiversidad y Gestión de } \\
\text { Ecosistemas }\end{array}$ & $\begin{array}{l}\text { Universidad Nacional Mayor de } \\
\text { San Marcos, Perú }\end{array}$ \\
\hline $\begin{array}{l}\text { Maestría en Conservación y Uso de } \\
\text { Biodiversidad }\end{array}$ & Pontificia Universidad Javeriana, Colombia \\
\hline Maestría en Altos Estudios Amazónicos & Pontificia Universidad Católica del Perú \\
\hline Maestría en Desarrollo Ambiental & Pontificia Universidad Católica del Perú \\
\hline $\begin{array}{l}\text { Maestría en Gestión de los Recursos } \\
\text { Hídricos }\end{array}$ & Pontificia Universidad Católica del Perú \\
\hline $\begin{array}{l}\text { Diploma Superior sobre Derecho y } \\
\text { Economía del Cambio Climático }\end{array}$ & FLACSO-Argentina \\
\hline $\begin{array}{l}\text { Magíster en Agroecología y Desarrollo } \\
\text { Sostenible }\end{array}$ & $\begin{array}{l}\text { Universidad Autónoma Gabriel René } \\
\text { Moreno, Bolivia }\end{array}$ \\
\hline Maestría en Tecnologías Ambientales & $\begin{array}{l}\text { Universidad Federal Tecnológica de Paraná, } \\
\text { Brasil }\end{array}$ \\
\hline Maestría en Biotecnología Industrial & Universidad Positivo, Brasil \\
\hline
\end{tabular}


En el caso de los doce programas de posgrado identificados ofrecidos por América del Norte, Europa u Oceanía, solo tres tienen un enfoque disciplinario y nueve tienen un enfoque interdisciplinario. Las maestrías que poseen un enfoque de especialización disciplinaria son las que tratan temas como gestión ambiental y gestión de la bioeconomía; estas maestrías son las siguientes: Maestría en Gestión en Bioeconomía de la Universidad de Lovaina en Bélgica, la Maestría en Gestión Ambiental para Negocios de la Universidad de Cranfield del Reino Unido y la Maestría en Gestión Ambiental de la Universidad de Queensland en Australia. Sin embargo, nueve maestrías tienen una visión interdisciplinaria, las cuales, tienen la orientación en temas de bioeconomía, desarrollo sostenible, gestión ambiental y medio ambiente y recursos naturales. Estas son:

\section{Cuadro 2. Otras maestrías con enfoque interdisciplinario}

\begin{tabular}{ll}
\hline \multicolumn{1}{c}{ Maestría } & \multicolumn{1}{c}{ Universidad } \\
\hline $\begin{array}{l}\text { Maestría en Gestión de la Bioeconomía, } \\
\text { Innovación y Gobernanza }\end{array}$ & Universidad de Edimburgo, Escocia \\
Maestría en Bioeconomía & Universidad de Hohenheim, Alemania \\
Maestría en Sostenibilidad e Innovación & HEC de París \\
Social & (Escuela de Estudios Comerciales) \\
Maestría en Desarrollo Sostenible & Universidad de Uppsala y la Universidad \\
& Sueca de Ciencias Agrícolas, Suecia \\
$\begin{array}{l}\text { Maestría en Medio Ambiente y Desarrollo } \\
\text { Sostenible }\end{array}$ & Escuela de Negocios de Adam Smith de la \\
Máster en Gestión Ambiental, Energía y & Universidad de Glasgow, Reino Unido \\
Responsabilidad Social Corporativa & Universidad de Bocconi, Italia \\
$\begin{array}{l}\text { Maestría en Ciencias en Estudios de Gestión } \\
\text { de Recursos y Medio Ambiente }\end{array}$ & Universidad de British Columbia, Canadá \\
Maestría en Medio Ambiente y Recursos & Universidad de Stanford, EE.UU. \\
Maestría en Medio Ambiente y Desarrollo & Escuela de Economía y Ciencia Política de \\
\hline
\end{tabular}

Fuente: Páginas web de programas de maestrías. Elaboración propia.

De la muestra de 36 maestrías, los programas orientados a la formación en biotecnología en general son disciplinarios, poseen cursos con alta especialización en tecnología molecular. Asimismo, la temática de los cursos de los programas orientados a la formación de gestión ambiental y gestión de energía 
está dirigida a temas de administración, contabilidad, gestión y economía sobre todo para la aplicación de los estudios desde la perspectiva del sector empresarial.

Las maestrías del exterior sobre bioeconomía, generalmente tienen cursos orientados en las áreas de economía, biotecnología, gestión y producción empresarial. La temática de los cursos en economía de esta rama es poco estructurada; proponen cursos básicos de economía, metodologías como optimización y econometría, funcionamiento de mercados y comercio. Por otra parte, los cursos de biotecnología están orientados a fabricar productos y mercancías con herramientas biotecnológicas, ya sean productos agrícolas, farmacéuticos o industriales. Asimismo, los cursos de gestión son cursos de innovación empresarial, análisis de costos, etc.

Por último, en el caso de los programas orientados a la formación de desarrollo sostenible, medio ambiente, recursos naturales y algunos programas de gestión ambiental, el área temática de sus planes de estudio incluye generalmente cursos de economía, gestión, biología, derecho y algunos con énfasis en el sector agrario. Es decir, que estos programas tienen una visión complementaria e interdisciplinaria. Así:

- Los cursos de economía tratan definitivamente el concepto de principios básicos de economía y desarrollo sostenible. Igualmente, proponen cursos de comercio, economía pública y desarrollo regional.

- Los cursos de gestión están básicamente orientados a la gestión de recursos naturales, ya sea recursos naturales, hídricos, biodiversidad, medio ambiente. Sin embargo, en las maestrías de gestión ambiental se incluyen cursos de responsabilidad empresarial.

- Los cursos de biología generalmente son para que los estudiantes conozcan cómo funciona la vida en el planeta. Es por eso que muchas de las maestrías ofertan cursos de biología y en especial ecología, la cual forma parte de cursos claves. Asimismo, existen cursos orientados directamente conocer la dinámica del medio ambiente y la economía.

- Los cursos orientados a la rama de derecho buscan que el estudiante conozca la legislación ambiental de su país, así como parte de la legislación sobre medio ambiente a nivel mundial. Por último, hay cursos en las maestrías orientados al desarrollo agrario sostenible, por lo cual que tienen énfasis en el biocomercio, en la agricultura ecológica y fomento de agronegocios. 


\begin{tabular}{|c|c|c|c|c|c|c|c|c|c|c|c|c|c|c|c|}
\hline & : & & 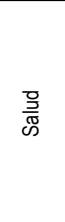 & & & & 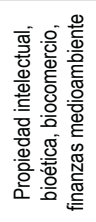 & 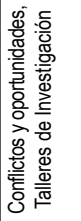 & & 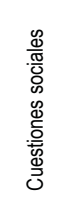 & $\begin{array}{l}\frac{\pi}{2} \\
\frac{0}{0} \\
\frac{0}{2} \\
\frac{2}{2}\end{array}$ & & & 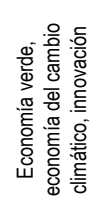 & \\
\hline & 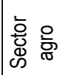 & & $x$ & $\times$ & & & $\times$ & & $\times$ & & $\times$ & & & & $\times$ \\
\hline & 灾 & & & & & & & & & $\times$ & $\times$ & & & & \\
\hline "s & 这 응 & & & & & & $\times$ & & $\times$ & & $\times$ & & $\times$ & $\times$ & \\
\hline 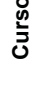 & \begin{tabular}{|l|}
\multicolumn{1}{|c}{} \\
0 \\
0
\end{tabular} & & & & & & $\times$ & $\times$ & & & $\times$ & & & & $x$ \\
\hline & \begin{tabular}{|l|l} 
\\
\end{tabular} & & $\times$ & & & & $\times$ & & & & $\times$ & & & $x$ & \\
\hline & 응 $\frac{\pi}{3}$ & & & & & & & & $\times$ & & & & $\times$ & & $\times$ \\
\hline & $\begin{array}{l}\dot{0} \\
\text { 음 } \\
\text { 응 }\end{array}$ & $\times$ & & $\times$ & $\times$ & & & & & & & & & & \\
\hline & 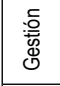 & & & & & & $\times$ & $\times$ & & $\times$ & $\times$ & & & $\times$ & \\
\hline & 嗃 & & & & & & $\times$ & & $\times$ & $\times$ & $\times$ & & $\times$ & $\times$ & $\times$ \\
\hline$\stackrel{\circ}{\stackrel{5}{\circ}}$ & 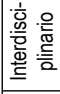 & & $\times$ & & & $\times$ & $\times$ & $x$ & $\times$ & $\times$ & $\times$ & $\times$ & $\times$ & $\times$ & $\times$ \\
\hline யี & 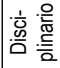 & $\times$ & & $\times$ & $\times$ & & & & & & & & & & \\
\hline$\frac{\overleftarrow{J}}{\frac{\delta}{w}}$ & 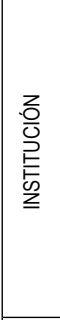 & 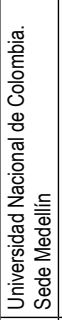 & 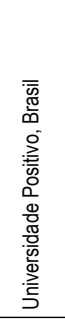 & 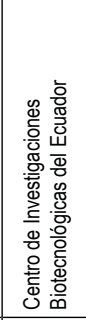 & 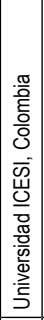 & 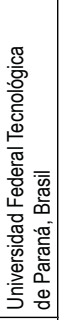 & 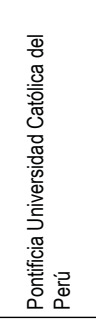 & 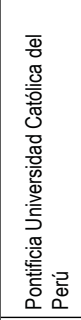 & 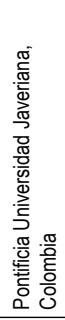 & 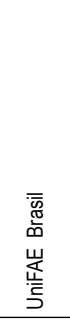 & 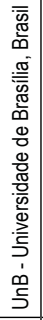 & 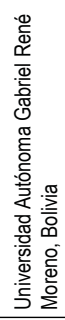 & 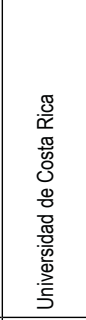 & 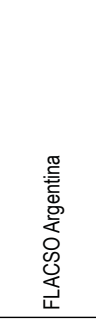 & 吾 \\
\hline 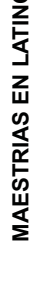 & 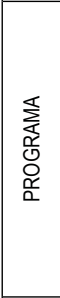 & 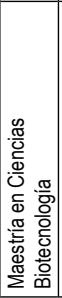 & 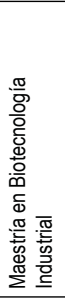 & 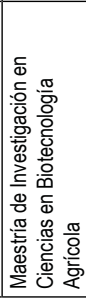 & 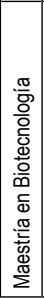 & 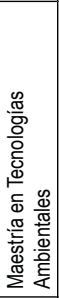 & 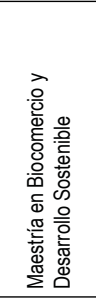 & 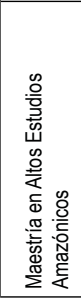 & 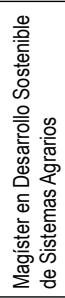 & 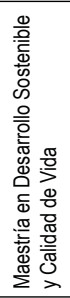 & 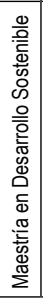 & 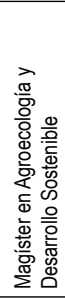 & 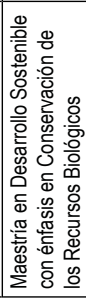 & 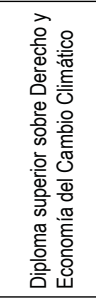 & 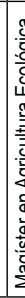 \\
\hline & 竎 & \multicolumn{5}{|c|}{ 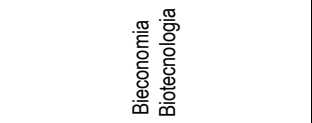 } & \multicolumn{9}{|c|}{ 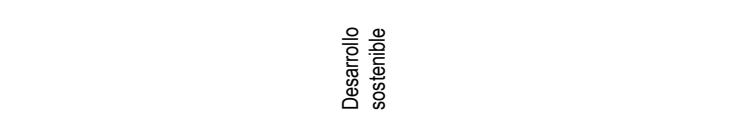 } \\
\hline
\end{tabular}




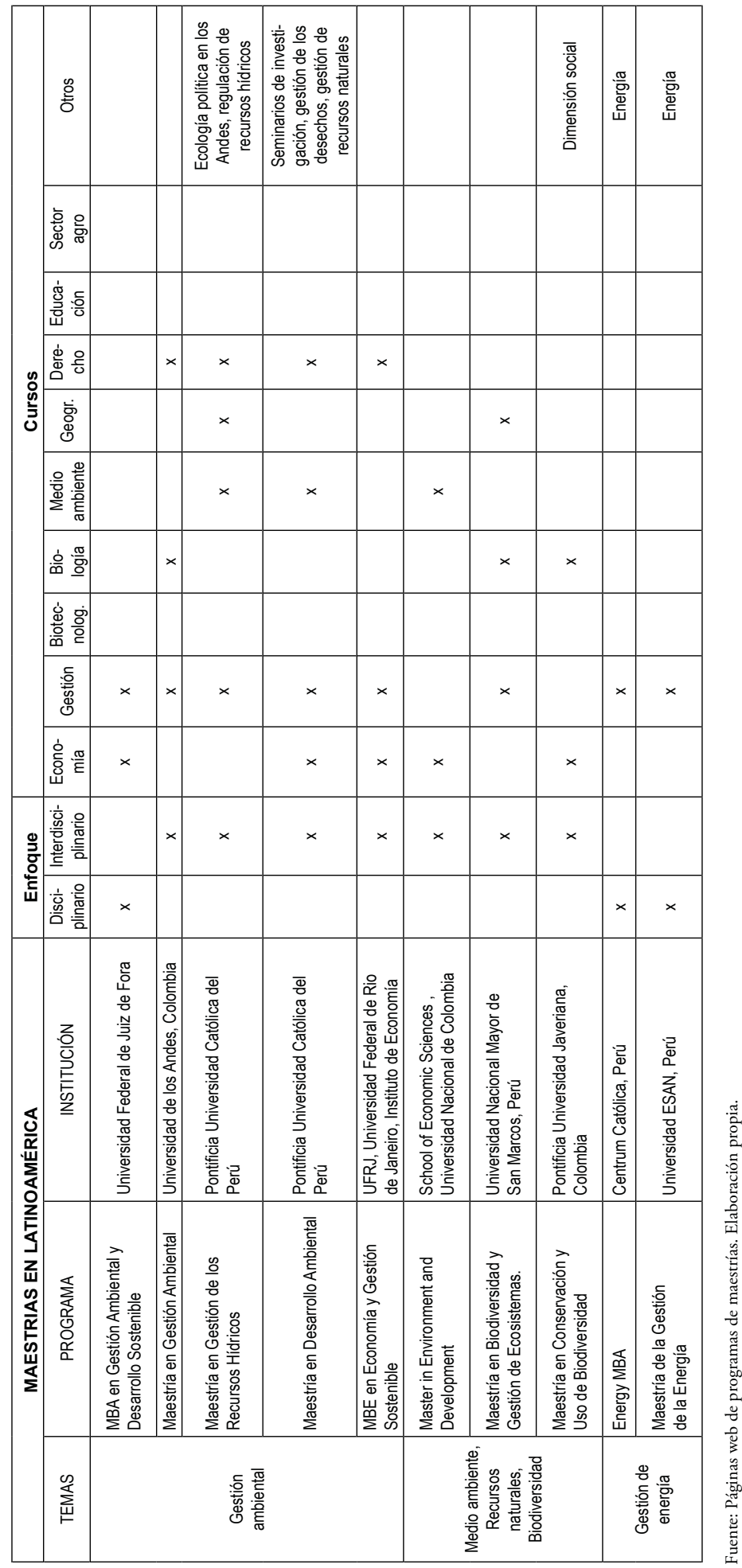




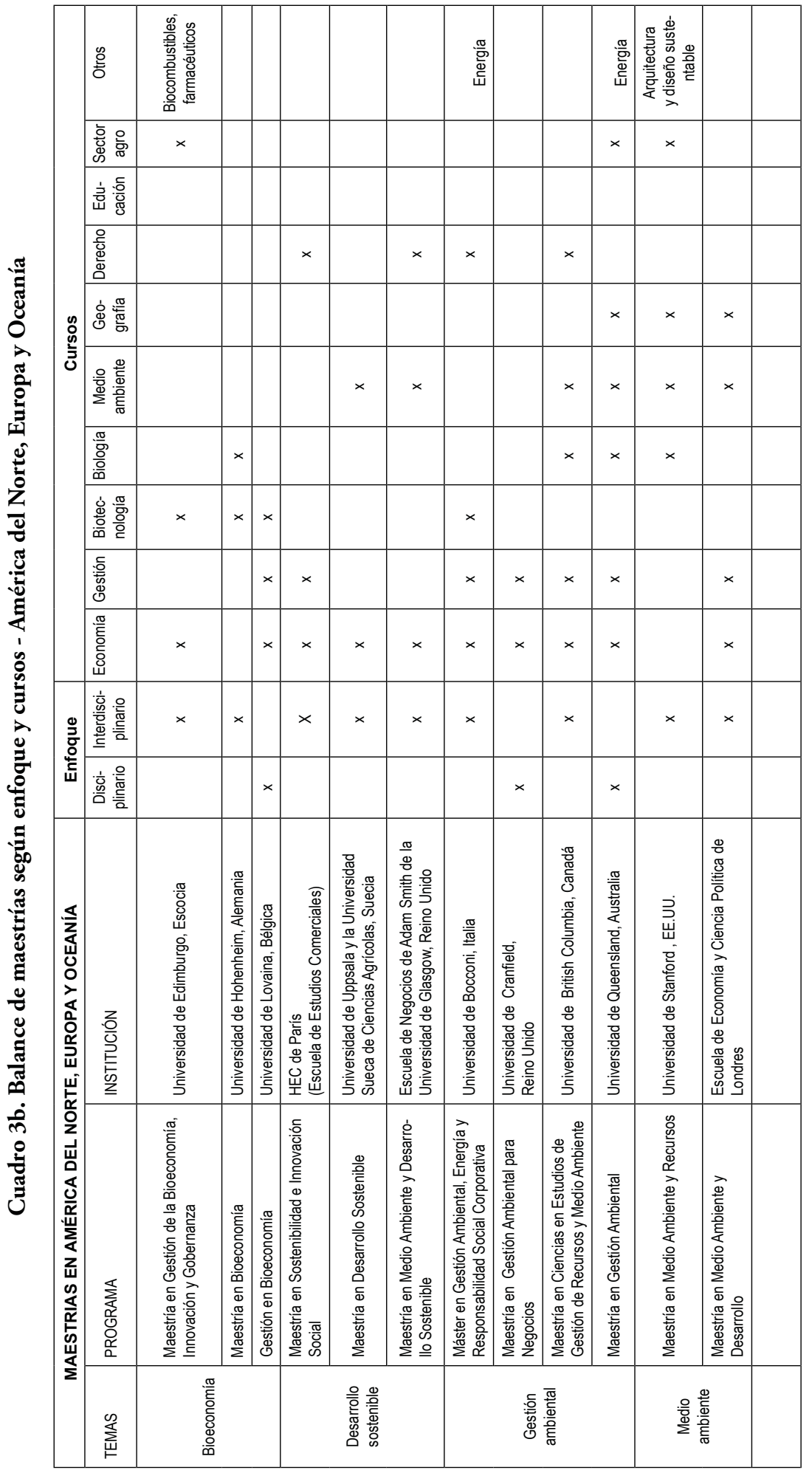

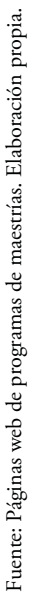




\section{Balance comparativo desde la Maestría en Biocomercio y Desarrollo Sostenible}

Primero se presentará la definición de biocomercio para poder contrastar con los enfoques de otras maestrías:

El concepto de biocomercio se refiere al conjunto de actividades producción, procesamiento y comercialización de bienes y servicios que son derivadas de la biodiversidad, estas actividades deben ser ejecutadas bajo criterios de sostenibilidad ambiental, social y económica debido a que son partes indesligables del desarrollo sostenible (UNCTAD, 2007, p. 1).

Es por eso, que el plan de Estudios de la Maestría de Biocomercio y Desarrollo Sostenible de la PUCP contiene cursos orientados a formar profesionales que puedan aplicar el tema de la sostenibilidad a nivel ambiental, social y económico. Asimismo, sus cursos principales están orientados a la articulación productiva, la propiedad intelectual y bionegocios.

Sobre la base de la clasificación temática realizada en las secciones 2 y 3 , se puede llegar a las siguientes observaciones comparativas respecto a la Maestría de Biocomercio y Desarrollo Sostenible.

Las maestrías con contenidos semejantes son generalmente las que trabajan el tema de desarrollo sostenible dentro de su contenido teórico. Sin embargo, las maestrías que tienen un contenido complementario son aquellas que se orientan al tema de bioeconomía, gestión ambiental, conservación de recursos naturales, gestión de energía. Son tres maestrías de Latinoamérica que tienen un enfoque muy parecido al de la maestría en Biocomercio y una de Europa. Trabajan a lo largo de su plan de estudios los temas de desarrollo sostenible, con cierto énfasis en los recursos agrícolas:

La Maestría en Desarrollo Sostenible de Sistemas Agrarios de la Pontificia Universidad Javeriana de Colombia, propone un enfoque interdisciplinario que incorpora el fomento con base científica de sistemas productivos agropecuarios sostenibles. Dentro del plan de estudios, los conceptos científicos y teóricos de sostenibilidad, ecología, biodiversidad y el enfoque agrario son los temas principales. De la misma forma, propone cursos para entender la economía rural y ambiental. Es así, que esta maestría tiene contenidos similares y convergen con los desarrollados por la Maestría en Biocomercio y Desarrollo Sostenible.

La Maestría en Agricultura Ecológica de la Universidad Nacional de Costa Rica forma en temas de análisis de los sistemas de producción y busca que los estudiantes generen opciones de solución a los problemas del impacto de la 
producción agrícola sobre el ambiente. Es decir, trata de que los profesionales se orienten a aplicar y promover la producción agrícola sostenible de manera multidimensional (social, económica, ecológica y ambiental). Sin embargo, si bien buena parte de sus cursos están orientados al concepto de sostenibilidad, la otra parte está orientada a los aspectos teóricos de la producción agrícola.

La Maestría de Desarrollo Sostenible con énfasis en Conservación de los Recursos Biológicos de la Universidad de Costa Rica busca que los profesionales puedan proponer solución de los problemas ambientales, la planificación urbana y el desarrollo sostenible, de esta forma, los cursos principales de su plan de estudios son los orientados a temas de desarrollo sostenible, biodiversidad y sociedad.

Del mismo modo, la Maestría en Desarrollo Sostenible de la Universidad de Uppsala y la Universidad de Ciencias Agrícolas de Suecia propone un enfoque interdisciplinario; en el cual, el contenido de sus cursos están orientados a dar una buena base en temas de desarrollo sostenible. Estas dos maestrías representan ejemplos de enfoques similares al que presenta la Maestría en Biocomercio y Desarrollo Sostenible, dado que brindan un debido énfasis a la formación en el concepto de sostenibilidad.

Por otro lado, las maestrías con enfoques complementarios al de la Maestría en Biocomercio y Desarrollo Sostenible, son aquellas que forman estudiantes en los temas de bioeconomía, gestión ambiental, medio ambiente, recursos naturales y gestión de energía. A continuación se presentará algunos ejemplos de estas maestrías.

En el tema de bioeconomía dos maestrías tienen miradas diferentes pero complementarias al de la Maestría en Biocomercio y Desarrollo Sostenible de la PUCP. La Maestría de Investigación en Ciencias en Biotecnología Agrícola del Centro de Investigaciones Biotecnológicas del Ecuador y la Maestría en Gestión de la Bioeconomía, Innovación y Gobernanza de la Universidad de Edimburgo en Escocia tienen la especial orientación de dotar de conocimiento sobre biotecnología con énfasis en la agricultura a los profesionales debido a que representa una herramienta potencial en el sector agrícola. Es decir, la biotecnología permite conocer los recursos genéticos que la biodiversidad contiene. Por este motivo, este tema y en especial estas maestrías representan un enfoque complementario al programa de Biocomercio.

En el tema de gestión ambiental, por ejemplo, los enfoques de Maestría en Gestión Ambiental de la Universidad de los Andes de Colombia y la Maestría en Gestión Ambiental de la Universidad de Queensland de Australia representan cierta complementariedad al enfoque de la Maestría de Biocomercio de la PUCP. Por un lado, la Maestría de la Universidad de los Andes busca formar 
estudiantes en gestión socioambiental y gerencia estratégica. Y, por el otro, la Maestría de la Universidad de Queensland propone un enfoque interdisciplinario de gestión ambiental que incluye el manejo sostenible sector por sector como minería, recursos naturales y marinos, agricultura, etc.

En el tema de conservación de medio ambiente y recursos naturales, existen dos maestrías que tienen propuestas complementarias al Programa de Posgrado de Biocomercio de la PUCP. Primero, la Maestría en Biodiversidad y Gestión de Ecosistemas de la Universidad Nacional Mayor de San Marcos en Perú es una propuesta que busca formar tanto en teoría como en la práctica a profesionales en el área de biodiversidad, que propone impulsar actividades en empresas, organizaciones e instituciones que desarrollen inventarios de la biodiversidad, desarrollo de planes territoriales, auditoría ambiental, diseño y gestión de áreas protegidas.

Asimismo, la Maestría en Conservación y Uso de Biodiversidad de la Pontificia Universidad Javeriana de Colombia tiene el objetivo de formar profesionales con base sólida sobre los enfoques, conceptos y métodos en el campo de la conservación y uso de la biodiversidad. Por lo tanto, los profesionales que cursen estas maestrías tendrán formación para el manejo y gestión de la biodiversidad.

Por último, en el tema de gestión de energía, se identificaron dos maestrías en el Perú complementarias al enfoque del Programa de Biocomercio y Desarrollo sostenible, ya que la gestión de energía eléctrica, de hidrocarburos y gas es un tema de suma importancia en el medio ambiente. Estas maestrías son el MBA en Energía de Centrum Católica y la Maestría de la Gestión de la Energía de la Universidad ESAN.

Con relación a los programas que ofrece la Escuela de Posgrado de la PUCP (Maestría en Altos Estudios Amazónicos, Maestría en Desarrollo Ambiental y Maestría en Gestión de Recursos Hídricos), se puede observar que aborda un amplio espectro de los estudios ambientales desde una aproximación interdisciplinaria. Es de mencionar que, estas maestrías junto a la Maestría en Biocomercio, son también programas de investigación que incluyen programas de tutorías y seminarios para el desarrollo de estas actividades.

Desde estos programas se propone incentivar el estudio de dinámicas de la sostenibilidad en el manejo de los recursos de la biodiversidad, teniendo como valor agregado las consideraciones culturales en el enfoque interdisciplinario que promueven. Es así que el perfil del estudiante está diseñado para profesionales de entidades públicas, privadas y organizaciones no gubernamentales relacionados con el aprovechamiento y gestión de la biodiversidad y en temas de desarrollo sostenible. 
Una de las ventajas que la PUCP ofrece la posibilidad de la articulación con otras maestrías, dando una enseñanza integral, donde los alumnos pueden acceder a la enseñanza de cursos de otros planes de estudios, de acuerdo al interés del alumno.

Esa complementariedad entre los programas brinda la posibilidad de ofrecer un área común, con cursos que brindan tanto especialización como la posibilidad de combinar diferentes materias según la experiencia y objetivos del estudiante. De esta manera la oferta de la PUCP se convierte en una alternativa interesante dado el mapeo de programas que se ha presentado en el presente documento. Asimismo, brindan una visión integral que es signo distintivo de la PUCP.

De esta manera, es de destacar que la Maestría en Biocomercio ofrece una oferta académica de tipo profesional sobre el sistema de biocomercio bajo la premisa de que es necesario articular la sostenibilidad económica, social y ambiental como partes indesligables del desarrollo sostenible.

El mayor valor agregado de este programa se centra en los cursos relacionados con planificación estratégica para el desarrollo sostenible, finanzas medioambientales y de desarrollo, comercio internacional y biodiversidad, cursos sobre los principios del biocomercio, y desarrollo de bionegocios.

Actualmente, la Maestría en Biocomercio y Desarrollo Sostenible está introduciendo nuevos cursos que buscan cubrir a profundidad nuevos temas, estos son: Política y legislación ambiental, Economía del cambio climático y desarrollo sostenible, Recursos energéticos renovables, y Valoración de la biodiversidad y huella de carbono. Asimismo, está emprendiendo una serie de acciones que buscan promover la internacionalización de la maestría e investigación, mediante creación de convenios, módulos cortos con profesores extranjeros, conferencias y seminarios con expertos nacionales e internacionales.

\section{Conclusión}

Finalmente se puede concluir que de los programas analizados, las maestrías que tratan el tema de desarrollo sostenible, medio ambiente y recursos naturales están casi siempre orientadas a tener un enfoque interdisciplinario. No obstante, la mayoría de los programas de posgrado orientados a temas de bioeconomía, biotecnología y gestión ambiental o de energía tienden a ser especializaciones disciplinarias.

Asimismo, existen áreas temáticas donde la cooperación sería beneficiosa entre instituciones. En particular: biocomercio, cambio climático y propiedad 
intelectual son áreas compartidas. Encontramos que lo más valioso no está en las materias estructurales o básicas, sino en la oferta de cursos optativos de los otros programas.

Ante esto la cooperación puede permitir expandir la oferta exportable y la internacionalización de los programas. Adicionalmente, entre los programas interdisciplinarios, los perfiles de formación y de los alumnos de los programas tienen puntos de contacto.

En efecto, la Maestría en Biocomercio de la PUCP está destinada a profesionales de las ciencias sociales, ciencias naturales y de gestión, entre otros; que requieran un conocimiento transversal del desarrollo sostenible para aplicación en sus respectivos campos laborales.

La colaboración entre instituciones puede enriquecerse no solo por el intercambio estudiantil, sino también por el intercambio docente y proyectos de investigación conjuntos que aporten a la generación de nuevos conocimientos, herramientas para el desarrollo de políticas y capacidades analíticas para la capacitación, análisis y evaluación así como brindar una formación más amplia, profunda y actualizada a aquellos profesionales que pretendan ampliar sus perspectivas laborales o fortalecer las herramientas teóricas y prácticas para un desempeño más eficiente y sofisticado en propiedad intelectual.

\section{REFERENCIAS BIBLIOGRÁFICAS}

Cosbey, A. (2011) Trade, Sustainable Development and a Green Economy: Benefits, Challenges and Risks. En UNDESA, UNEP, UNCTAD, The Transition to a Green Economy: Benefits, Challenges and Risks from a Sustainable Development Perspective (pp. 40-68). Disponible en https:// sustainabledevelopment.un.org/index.php?page=view\&type=400\&nr=13 $1 \&$ menu $=1515$

Da Motta, P. \& Polónia, S. (2013). Políticas e impacto del crecimiento verde en América Latina. Serie Crecimiento Verde e Inclusivo 89. Red Latinoamericana de Política Comercial (LATN).

Fairlie, A. (2013). Crecimiento verde y biocomercio: una mirada andina. Lima: Pontificia Universidad Católica del Perú, Departamento de Economía.

Hallegatte, S., Heal, G., Fay, M. \& Treguer, D. (2012). From Growth to Green Growth - A Framework. NBER Working Paper 17841. Disponible en http://www.nber.org/papers/w17841.pdf

Khor, M. (2011). Challenges of the Green Economy Concept and Policies in the Context of Sustainable Development, Poverty and Equity. En UNDESA UNEP, UNCTAD, The Transition to a Green Economy: Benefits, Challenges 
and Risks (pp. 69-97). Disponible en https://sustainabledevelopment. un.org/index.php?page $=$ view\&type $=400 \& n r=131 \&$ menu $=1515$

Martínez Alier, J. \& Roca Jusmel, J. (2001). Economía ecológica y politica ambiental. México: FCE.

Neumayer, E. (2003). Weak versus Strong Sustainability: Exploring the Limits of Two Opposing Paradigms. Northampton: Edward Elgar.

Ocampo, J. A. (2011). The Transition to a Green Economy: Benefits, Challenges and Risks from a Sustainable Development Perspective. Summary of Background Papers. En UNDESA, UNEP, UNCTAD, The Transition to a Green Economy: Benefits, Challenges and Risks (pp. 3-15). Disponible en https://sustainabledevelopment.un.org/index.php?page=view\&type $=400$ $\& n r=131 \&$ menu $=1515$

Organization for Economic Co-operation and Development (OECD) (2011). Towards Green Growth. París: OECD Publishing. http://dx.doi. org/10.1787/9789264111318-en

UNCTAD - Conferencia de las Naciones Unidas sobre Comercio y Desarrollo (2007). Informe sobre el comercio y el desarrollo, 2007. Ginebra: Cooperación Regional para el Desarrollo. Disponible en http://unctad.org/es/docs/ tdr2007_sp.pdf 